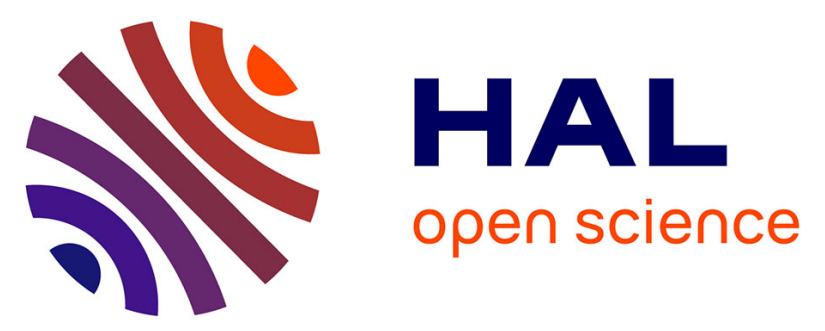

\title{
L'usure des travailleurs vieillissants à l'épreuve de la nationalité. Le cas des migrants de Tunisie en région parisienne dans la seconde moitié du XXe siècle
}

\author{
A.-S. Bruno
}

\section{- To cite this version:}

A.-S. Bruno. L'usure des travailleurs vieillissants à l'épreuve de la nationalité. Le cas des migrants de Tunisie en région parisienne dans la seconde moitié du XXe siècle. Psychologie du travail et des organisations, 2019, 25, pp.6 - 15. 10.1016/j.pto.2018.06.002 . hal-03486381

\section{HAL Id: hal-03486381 \\ https://hal.science/hal-03486381}

Submitted on 20 Dec 2021

HAL is a multi-disciplinary open access archive for the deposit and dissemination of scientific research documents, whether they are published or not. The documents may come from teaching and research institutions in France or abroad, or from public or private research centers.
L'archive ouverte pluridisciplinaire HAL, est destinée au dépôt et à la diffusion de documents scientifiques de niveau recherche, publiés ou non, émanant des établissements d'enseignement et de recherche français ou étrangers, des laboratoires publics ou privés.

\section{(ㄷ)(1) $\$$}

Distributed under a Creative Commons Attribution - NonCommercial| 4.0 International 


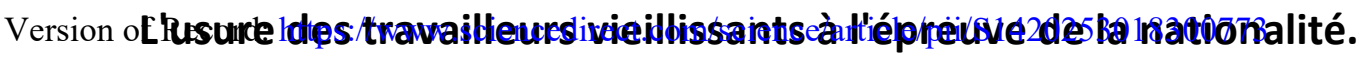

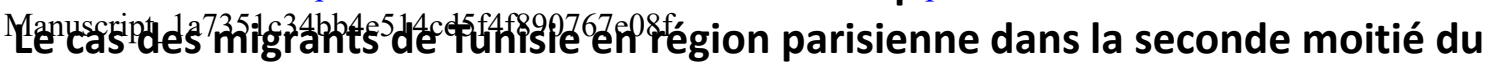
$20^{\mathrm{e}}$ siècle

The wear and tear of ageing workers through the prism of nationality. The case of Migrants from Tunisia in the Paris region in the second half of the 20th century

Anne-Sophie Bruno, maître de conférences en histoire contemporaine, Université Paris 1Panthéon Sorbonne, Centre d'histoire sociale du $20^{\mathrm{e}}$ siècle (UMR 8058, CNRS-Université Paris 1)

Correspondant : Anne-Sophie Bruno, 06-19-92-51-98, Anne-Sophie.Bruno@univ-paris1.fr

Pas de conflits d'intérêt 
Résumé.

Si les travailleurs étrangers sont exposés à des conditions de travail et d'emploi dégradées, ils ne sont pas nécessairement affectés par une surmortalité particulière. Par l'analyse des dispositifs de protection sociale et de données biographiques, l'article montre que cette situation paradoxale résulte de la combinaison de plusieurs facteurs qui contribuent à une invisibilité relative des problèmes de santé rencontrés par les travailleurs étrangers.

En effet si les travailleurs étrangers constituent une main-d'œuvre sur-sélectionnée, les effets à long terme de leurs conditions de travail restent méconnus, en raison des représentations particulières dont ils font l'objet mais aussi de la segmentation du marché du travail, qui induit des trajectoires différenciées du point de vue de la santé et de sa prise en charge.

\section{Abstract}

If foreign workers are exposed to degraded working and working conditions, they are not necessarily affected by a particularly increased mortality. By analyzing social protection schemes and biographical data, the article shows that this paradoxical situation results from the combination of several factors that contribute to the relative invisibility of the health problems faced by foreign workers.

Indeed, if foreign workers constitute an over-selected workforce, the long-term effects of their working conditions remain unknown, because of the particular representations they receive, but also because of the segmentation of the labor market, which induces differentiated trajectories from the point of view of health and its management.

Mots-clés: inégalités de santé, trajectoires professionnelles, travailleurs étrangers, usure au travail

Key words: health inequalities, career paths, foreign workers, job-related wear and tear. 
La question des inégalités de santé est devenue cruciale depuis l'entrée dans l'ère industrielle, qui a vu se creuser les écarts de mortalité entre les différentes catégories socioprofessionnelles. Ces écarts apparaissent comme particulièrement forts en France où, en 1990, le risque de décéder entre 45 et 59 ans est 1,71 fois plus élevé pour les ouvriers que pour les cadres et professions libérales (Goldberg et al., 2003) ; en 2018 l'écart d'espérance de vie entre les $5 \%$ plus aisés et les $5 \%$ les moins aisés étaient toujours de 13 ans pour les hommes (Blanpain, 2018). De même, pour les hommes, l'écart du risque de mortalité prématurée (de 45 à 59 ans) entre les ouvriers et ceux exerçant une profession non manuelle est environ deux fois plus élevée qu'en Italie, en Espagne, au Portugal, en Suède ou au Danemark. Les années 2000 ont ainsi vu se développer les enquêtes sur les inégalités de santé, qui ont souligné le rôle des facteurs professionnels dans leur apparition (Leclerc et al., 2000). Dans cet ensemble, les inégalités liées à la nationalité ou à l'origine apparaissent toutefois comme particulièrement difficiles à cerner. Leur analyse se heurte notamment à un paradoxe: si les étrangers apparaissent comme particulièrement exposés à des conditions de travail et d'emploi dégradées, les études menées en population générale ne mettent pas en évidence de surmortalité particulière (Darmon \& Khlat, 2001, Khlat \& Courbage, 1995, Khlat, Sermet \& Laurier, 1998, Mizrahi \& Wait, 1993). L'hypothèse que j'explorerai ici est que cette situation paradoxale résulte de la combinaison de plusieurs facteurs qui contribuent tous à une invisibilité relative des problèmes de santé rencontrés par les travailleurs étrangers.

Nous verrons en effet que s'il s'agit d'une main-d'œuvre sur-sélectionnée à l'entrée sur le marché du travail (1), les effets à long terme de leurs conditions de travail restent largement méconnus, en raison notamment des représentations particulières dont font l'objet les travailleurs étrangers (2). Face à ce constat, l'approche longitudinale permet de dégager quelques éléments de réponse sur la construction des inégalités de santé en fonction de la nationalité (3).

\section{Une main-d'œuvre sur-sélectionnée sur un plan médical et professionnel}

L'impératif de sélection médicale et professionnelle des travailleurs migrants apparaît en même temps que la figure du travailleur immigré, dont il est l'un des éléments constitutifs (Bruno, 2004). Il a en effet pour but de s'assurer de l'employabilité immédiate de travailleurs étrangers perçus comme une main-d'œuvre d'appoint destinée à combler les espaces de travail laissés par les nationaux et dont la présence sur le territoire est de plus en plus étroitement subordonnée à leur utilité économique à mesure que le $20^{e}$ siècle avance. De ce fait, les premiers recrutements collectifs de travailleurs étrangers s'accompagnent de la mise en place de dispositifs de sélection, appliqués notamment aux Italiens recrutés par le Comité des Houillères à la Belle Epoque. La Première Guerre mondiale constitue également une étape décisive dans la mise en place de cette sélection médicale, à laquelle tous les travailleurs coloniaux sont soumis dès leur arrivée en France.

Loin de disparaître avec le retour à la paix, ces pratiques sont reconduites après guerre, à une plus large échelle. Les exigences de sélection médicale deviennent ainsi un élément central des conventions bilatérales d'émigration signées par la France avec un certain nombre de pays européens, comme l'Italie, la Pologne ou la Tchécoslovaquie, et ce de façon durable puisqu'elles continueront d'animer les conventions signées après 1945. 
Dans l'entre-deux-guerres, le contrôle de l'aptitude est ainsi obligatoire pour les étrangers recrutés de façon collective, qui représentent alors l'écrasante majorité des flux migratoires d'étrangers (50 000 des 60000 travailleurs immigrés introduits en 1929). Confiée à partir de 1924 à la Société générale d'immigration, émanation des principales organisations patronales de l'industrie et de l'agriculture, la sélection pratiquée à l'occasion de ces visites d'aptitude s'avère drastique : dans les années vingt, c'est un peu plus d'un tiers des Polonais et des Tchécoslovaques candidats à l'émigration qui se voient refuser le droit d'entrer en France pour raison d'inaptitude.

Ces dispositifs constituent la matrice du contrôle de l'aptitude médicale qui est généralisé à l'ensemble des travailleurs après 1945. Pour autant cette généralisation n'entraîne pas d'abandon des contrôles spécifiques pour les travailleurs étrangers, qui sont désormais pris en charge par le nouvel office public en charge de l'immigration. On assiste dès lors pour les étrangers à une juxtaposition des dispositifs de contrôle, qui, en l'absence d'une harmonisation des critères, sont source de décisions contradictoires et d'incompréhension, notamment de la part d'individus reconnus aptes par l'Office National d'Immigration, mais rejetés ensuite au moment de la visite d'embauche pratiquée par le médecin du travail.

Destiné à assurer la sélection d'une main-d'œuvre immédiatement utile, ce contrôle de l'aptitude des travailleurs étrangers repose sur des critères à géométrie variable, largement dépendants de l'état de la conjoncture. Selon les besoins de l'économie, les limites d'âge peuvent ainsi être abaissées ou relevées, de la même façon que la sévérité des critères varie selon le type de contrat, saisonnier ou permanent ${ }^{1}$. De ce point de vue, les employeurs apparaissent comme les principaux acteurs de la définition de ces critères d'aptitude, avant comme après la Seconde Guerre mondiale.

Ces formes institutionnalisées de contrôle médical, qui dessinent une forme de bonne santé conventionnelle, et institutionnalisée, viennent ainsi s'ajouter aux formes de sélection "spontanée " des plus riches et des mieux portants, observées en amont parmi les candidats à l'émigration; elles ne font ainsi que renforcer le healthy migrant effect souvent évoqué à propos des travailleurs migrants.

Si les travailleurs étrangers sont majoritairement recrutés pour effectuer des travaux pénibles, délaissés par les nationaux, la sélection drastique dont ils font l'objet à leur entrée sur le marché du travail permet sans doute de compenser cette surexposition au risque professionnel. Le traitement particulier qui leur est réservé pose donc la question des effets cumulés et différés des conditions de travail auxquels ils sont exposés. De ce point de vue, on se heurte à une relative invisibilité des risques spécifiques encourus par cette catégorie de main-d'œuvre.

\section{L'impossible mise en lumière des risques spécifiques aux travailleurs étrangers}

Cette invisibilité des risques spécifiques est en grande partie construite par le discours médical sur la santé des travailleurs étrangers. Jusqu'aux années 1960, l'intérêt porté par les revues de médecine du travail aux populations étrangères et coloniales est

\footnotetext{
$1 \quad$ A la fin des années 1950, les taux d'inaptitude observés chez les travailleurs espagnols et italiens sont de l'ordre de $10 \%$ pour les travailleurs permanents, contre $3 \%$ chez les saisonniers.
} 
faible $^{2}$; le discours médical y apparaît en outre comme ambivalent, cherchant à lutter contre des préjugés naturalisants dont il ne parvient toutefois pas à s'abstraire. Cette ambivalence caractérise notamment le traitement réservé au contrôle sanitaire aux frontières, préoccupation majeure concernant la santé de travailleurs perçus comme les vecteurs de maladies contagieuses et de maladies sexuellement transmissibles. Si les résultats des enquêtes concluent à la faible importance de ce risque, le dépistage des maladies contagieuses et exotiques à l'occasion des visites d'embauche apparaît néanmoins comme un passage obligé, et central, de la littérature médicale sur les travailleurs étrangers (Housset et al., 1965). De ce point de vue, la population coloniale algérienne, qui jouit de la nationalité française et qui de ce fait échappe à tout contrôle aux frontières, réactive les craintes de contagion (Colin, 1953) ; les thèses de médecine qui lui sont consacrées font dès lors une large part au risque de contagion par la tuberculose ${ }^{3}$ et aux infrastructures de surveillance médicale de cette population (Al Zerdoumi, 1952).

Au-delà du cas des travailleurs algériens et de la question de maladies infectieuses telles que la tuberculose, les capacités d'adaptation des travailleurs immigrés au milieu d'accueil font également l'objet de nombreux préjugés. Si la surexposition des travailleurs d'origine étrangère à des risques professionnels suscite des avis divergents, les considérations relatives à la pénibilité des postes occupés apparaissent comme marginales au regard des particularités biologiques ou comportementales attribuées aux travailleurs étrangers. Les stéréotypes de groupe sont ici légion, qu'il s'agisse du caractère superstitieux ou puéril des Nord-Africains (Colin, 1953), du sens aigu du droit dont ils font preuve, de la docilité caractéristique des originaires d'Afrique noire ou de l'anxiété et de la volubilité particulières des Espagnols (Housset et al., 1965). Lorsque la question des accidents du travail est évoquée, elle est fréquemment associée à une sensibilité particulière au risque de sinistrose, c'est-à-dire à une majoration inconsciente des effets de l'accident par la victime, débouchant sur une psychose revendicative.

Cette invisibilité des risques spécifiques liés aux postes de travail qu'occupent les travailleurs d'origine étrangère est toutefois battue en brèche par les mouvements de contestation de la fin des années 1960 et du début des années 1970, au sein desquels les femmes et les étrangers font irruption, dénonçant les effets nocifs des conditions de travail sur leur santé. Ces mobilisations qui mettent au centre de leurs revendications les questions de santé et de conditions de travail trouvent alors un appui décisif de la part de syndicalistes et de professionnels de la santé au travail (Pitti, 2009 et 2010). A la faveur de ce mouvement, les articles sur les étrangers se multiplient dans la presse spécialisée de la médecine du travail et abordent désormais la question de la santé au travail par les conditions et postes de travail, largement occultée jusque-là.

Né dans le sillage des événements de mai $68^{4}$, le Comité Médical et Médico-social d'Aide aux Migrants joue un rôle essentiel dans la production d'un discours sur la santé des étrangers, qui s'impose progressivement et se diffuse au-delà de la profession médicale dans

\footnotetext{
2 Au cours des années 1950 et 1960, les Archives des maladies professionnelles (désormais AMP) ne consacrent en effet que trois articles et trois recensions de thèse de médecine aux travailleurs migrants.

3 Bresson J.-R., Le problème de la tuberculose pulmonaire du Nord-Africain, thèse de médecine, Lyon, 1951-1952 (voir compte rendu dans $A M P, 1953,14 / 1,96-97)$, ainsi que la thèse de R. Diacre (Paris, 1949), citée par Colin, 1953). La morbidité des Nord-Africains dans la métropole, AMP, 14/6, 594-601.

$4 \quad$ Constitué de façon informelle en 1968, le Comité est créé officiellement en 1970 (voir Bulletin Migrations Santé, $\left.\mathrm{n}^{\circ} 1,1971\right)$.
} 
les années 1970-1980. C'est à lui que le MRAP s'adresse en 1972 pour éditer un guide sur la santé des étrangers, devenu rapidement un classique cité dans toutes les études (Comité médical et médico-social d'aide aux migrants, 1972). C'est également le Comité qui est sollicité sur les questions de santé lors du colloque organisé par la CFDT sur les travailleurs étrangers en 1979. Deux de ses membres sont également les auteurs d'un rapport sur la santé des migrants commandé par le ministre des Affaires sociales en 1984 (Gentilini, Brücker \& de Montvalon, 1984). Son discours en triptyque distingue les maladies d'apport ou d'importation, les pathologies d'acquisition et les pathologies d'adaptation.

Pourtant, malgré la volonté de ces médecins de ne pas s'enfermer dans la défense des étrangers et d'intégrer la question immigrée dans une revendication plus générale autour de l'amélioration des conditions de travail (Montvalon, 1976), le discours accorde toujours plus de place aux pathologies spécifiques (parasitoses, dermites d'importation) et aux pathologies auxquelles les étrangers sont surexposés (tuberculose, MST) qu'aux pathologies professionnelles (dermatoses, maladies respiratoires non infectieuses...), pourtant beaucoup plus répandues. Ainsi, l'attention accordée à la tuberculose peut s'expliquer par la surexposition des étrangers, qui présentent un risque tuberculeux deux fois plus élevé que les nationaux (et même vingt à trente fois pour les originaires d'Afrique subsaharienne) du fait de primo-infections précoces plus fréquentes et d'infections contractées plus fréquemment dans la migration, du fait de leurs conditions de travail et de logement ; pour autant, la morbidité par tuberculose concerne moins d'un étranger sur mille et sa prise en charge médicale permet, à cette date, une bonne évolution de la maladie, sans commune mesure avec la morbidité et la mortalité des maladies respiratoires qui touchent les travailleurs du bâtiment exposés à l'amiante et à la poussière de silice (mésothéliome, asbestose, plaques pleurales et silicose). De la même façon, alors que les dermites d'apport représentent quelques centaines de cas par an, elles occupent une place centrale dans les recherches médicales sur la santé des travailleurs étrangers, loin devant les dermatoses professionnelles, qui affectent pourtant plusieurs milliers de personnes ${ }^{5}$.

Le renouvellement du discours médical bute ici sur le profil institutionnel des médecins qui s'expriment alors dans les congrès et les revues professionnelles et qui sont pour la plupart des spécialistes de santé publique, sous son versant maladies contagieuses. $A$ contrario, les médecins du travail du bâtiment donnent à voir, au travers de la Revue de Médecine du Travail, une vision concurrente, davantage fondée sur une analyse des pathologies professionnelles en lien avec les conditions de travail et les structures d'accueil : ils y décrivent la surexposition des étrangers aux pathologies masculines (cardiopathies, tuberculose, asthme, hernies et ulcères) très sensibles aux facteurs environnementaux, et notamment aux conditions de travail difficiles et aux fortes contraintes physiques (charges lourdes, expositions aux fumées et aux poussières, à la chaleur, au froid). L'attention que ces médecins du BTP portent aux conditions de travail peine toutefois à dépasser les contours de leur branche d'activité, avant que la crise ne vienne éclipser la question des conditions de travail, au profit des problèmes d'emploi.

Si la mobilisation des travailleurs étrangers autour des conditions de travail a eu une certaine résonance dans les milieux syndicaux et médicaux, les initiatives prises pour relayer la dénonciation des conditions de travail ont ainsi buté sur les difficultés à faire remonter et

\footnotetext{
5 Chaque année, près de 1500 dermatoses liées au ciment sont ainsi reconnues comme maladie professionnelle, les deux tiers d'entre elles touchant des travailleurs étrangers.
} 
à intégrer le discours propre à la santé au travail des étrangers dans une revendication plus générale intéressant l'ensemble des travailleurs.

Après ce court épisode de médiatisation, la santé des étrangers retombe alors dans une invisibilité prolongée puisque, jusqu'au début des années 1990, la variable de la nationalité n'est en effet mentionnée dans aucune grande enquête sur la santé et les conditions de travail. Certains médecins se donnent les moyens de construire des protocoles d'enquête rigoureux sur certaines sous-populations immigrées ${ }^{6}$, mais leurs résultats ne s'étendent jamais à l'ensemble de la population étrangère ${ }^{7}$. Quand les études existent, elles aboutissent à des conclusions parfois contradictoires : l'effet de la nationalité n'est pas clairement établi et dépend du point d'observation, selon qu'il s'agit de santé perçue ou d'état de santé fonctionnel (Jusot et al., 2008). En ce qui concerne la surexposition des étrangers aux accidents du travail, les médecins du travail soulignent eux-mêmes qu'ils ne peuvent pas dire grand-chose des différences d'exposition qu'ils observent (Perrin, 1976). Dans ces conditions, le recours à des données longitudinales permet en partie de contourner ces difficultés et de mieux saisir la façon dont on peut analyser la santé des travailleurs étrangers, et plus globalement celle de l'ensemble des travailleurs.

\section{Les apports de la démarche longitudinale}

L'analyse présentée dans cette section se fonde sur des données biographiques recueillies sur un échantillon de 193 individus nés en Tunisie entre le début des années 1910 et la fin des années 1930 et qui ont migré en région parisienne entre le début des années 1950 et le début des années 1970 (Bruno, 2010). La définition de cette population permet de comparer le devenir de populations françaises rapatriées en métropole après l'indépendance de la Tunisie à celui de populations étrangères - tunisiennes ou italiennes ou nées étrangères et devenues françaises avant ou après la migration en région parisienne. L'analyse longitudinale de leurs données de carrière s'adapte particulièrement bien à une étude de l'état de santé, qui se construit dans la durée, tout au long de la trajectoire familiale et professionnelle. Les données recueillies sur ces migrants de Tunisie ont en outre pour particularité de reposer sur un principe d'appariement qui permet de croiser des données individuelles de salariés du régime général parvenus à la retraite avec des données sur leurs espaces de travail, perçus par le biais des déclarations annuelles de salaires des entreprises dans lesquelles ont travaillé les actifs de l'échantillon étudié ${ }^{8}$. Leur exploitation permet ainsi d'explorer les vertus méthodologiques du recours aux données appariées employeurs-employés en santé et travail.

Si ces données ne portent pas à proprement parler sur l'état de santé des individus de mon échantillon, elles livrent néanmoins des informations utiles sur les mécanismes différenciés de l'usure au travail, par le biais des indicateurs de santé administratifs que sont

\footnotetext{
6 Voir notamment «Enquête sur l'absentéisme des Africains » (1979). Cahiers de Médecine Interprofessionnelle, 75, 5-19.

Le professeur M. Gentilini déplore ainsi que I'INSERM se soit « en pratique refusé à aborder le problème des travailleurs migrants » (de Montvalon, 1975).

$8 \quad$ Les données recueillies sur les entreprises qui ont occupé les salariés de notre échantillon permettent de reconstituer la nationalité et le genre de leurs collègues de travail, mais aussi la durée de présence dans l'entreprise et le type de postes occupés par l'ensemble des salariés de ces entreprises.
} 
le versement d'une allocation invalidité ou maladie et l'accès à la retraite anticipée au titre de l'inaptitude au travail.

Le traitement quantitatif des biographies collectives des migrants de Tunisie met en évidence le rôle de l'organisation du marché du travail dans la construction des inégalités de santé selon la nationalité. Au regard des résultats de l'analyse de régression logistique, les caractéristiques relatives à l'identité sociale des individus - genre, nationalité.... - ont peu d'influence sur la probabilité de connaître des problèmes de santé en fin de carrière; en revanche, les facteurs qui ont trait à la position professionnelle et à l'organisation du marché du travail (type d'emploi, secteur d'activité et segment du marché sur lesquels l'individu est positionné) ont un effet significatif.

Ainsi, le simple fait d'avoir occupé un emploi ouvrier au cours de sa carrière multiplie par quatre le risque d'avoir des problèmes de santé en fin de parcours : en raison de leur surreprésentation dans les emplois ouvriers, les étrangers - italiens ou tunisiens - de notre échantillon sont de ce fait plus exposés à ce risque que les Français originaires de Tunisie.

Les effets de durée dans les emplois ouvriers sont également perceptibles sur les fins de carrière des migrants de Tunisie. Le risque d'être déclaré inapte au moment de la retraite est plus grand lorsque l'individu a exercé longtemps un emploi ouvrier au cours de sa carrière : dans le modèle de régression, la variable "avoir exercé un emploi ouvrier" ne joue en effet un rôle significatif que lorsqu'elle est couplée au temps passé sur le marché du travail. Ce résultat rappelle que l'usure au travail (Cottereau, 1983), phénomène essentiellement ouvrier, est avant tout une affaire de durée, qui échappe par conséquent à une observation transversale faite à un instant $t$. II rejoint les conclusions de Catherine Omnès sur le profil particulier des femmes reconnues inaptes au travail à la même période, dont beaucoup sont des ouvrières à vie (Omnès, 2006).

Le segment de marché sur lequel le travailleur évolue joue également un rôle majeur dans les inégalités de santé au travail. C'est ce que montre l'analyse des fins de carrière des migrants de Tunisie à la lumière d'une typologie ternaire qui élargit celle développée dans les années 1970 par les tenants du dualisme du marché du travail, notamment par Piore et Doringer. Sur le marché du travail des natifs de Tunisie pendant les Trente Glorieuses, le segment primaire est constitué d'emplois relativement stables et bien rémunérés, correspondant aux entreprises du marché interne caractéristique d'une relation salariale de type fordiste, tandis que le segment secondaire est composé d'emplois instables et mal rémunérés, essentiellement dans le bâtiment et les travaux publics ou dans le nettoyage et le textile. Quant au segment intermédiaire, celui des petites entreprises, il offre une plus grande stabilité de la relation salariale que le secondaire, mais un plus faible niveau de rémunération que le primaire et correspond à bon nombre de petites entreprises du commerce et de la restauration. Sans surprise les étrangers de notre échantillon sont surreprésentés sur le segment secondaire, quand les hommes de nationalité le sont sur le segment primaire, laissant nombre de femmes de toutes nationalités travailler sur le segment intermédiaire.

L'importance des espaces de travail fait apparaître les dénominations fondées sur le métier ou le niveau de qualification comme insuffisantes: pour saisir la position socioéconomique d'un individu, il ne suffit pas de lui demander le métier qu'il exerce, il faut aussi lui demander où il travaille. L'agent d'entretien des collectivités territoriales et celui des sociétés privées de nettoyage ont certes en commun l'objet de leur activité - le nettoyage - 
et leur faible niveau de qualification. Mais ces caractéristiques habituellement perçues comme un bon indicateur de la position sociale ne rendent pas compte de la hiérarchie des positions sociales : l'ouvrier des grandes sociétés de nettoyage n'a ni la stabilité de l'emploi ni le niveau de salaire de l'agent municipal employé par la Ville de Paris. Dans ce dernier cas, le poste occupé donne lieu à un salaire décent et à une stabilité de l'emploi quand le premier type de poste signifie, pendant les Trente Glorieuses, changer cinq ou six fois d'employeur chaque année et percevoir au final un salaire de misère. Deux individus peuvent ainsi exercer le même métier, ou avoir une qualification équivalente, et ne pas avoir du tout la même vie, au travail comme en dehors. On peut même dire que cette hétérogénéité des espaces de travail constitue le principal facteur d'inégalités, en particulier salariales, pendant les Trente Glorieuses.

Ces trajectoires différenciées s'observent aussi en fin de carrière. La crise économique touche surtout les travailleurs vieillissants ou d'âge mûr. Au seuil de la retraite, moins de la moitié des natifs de Tunisie appartenant à la cohorte 1913-1915 est encore en emploi à 64 ans. Dès les années 1970, parmi les salariés qui ont atteint l'âge de 59 ans, un individu sur cinq a quitté le marché du travail. Ce mouvement de réduction de l'emploi des travailleurs âgés n'est pas propre aux individus de notre échantillon. En 1975, en France, le taux d'activité des 60-64 ans n'est plus que de $54 \%$ et près d'un homme sur cinq est sorti du marché du travail entre 55 et 59 ans (Minni \& Topiol, 2004, Guillemard, 1993). Après l'abaissement de l'âge légal de la retraite à 60 ans en 1982, le seuil de la vieillesse s'abaisse brusquement : le déclin de l'activité commence désormais dès 55 ans. À 59 ans, près de $60 \%$ des individus des cohortes nées après 1923 sont déjà sortis de l'emploi : si le seuil de la vieillesse a été abaissé, la proportion de travailleurs âgés sortis du marché du travail dans les années précédant leur retraite est donc restée inchangée. L'âge légal de la retraite apparaît ainsi comme un élément déterminant dans le calendrier des sorties d'emploi. Les départs des travailleurs vieillissants, étrangers comme nationaux, sont moins liés à une usure naturelle qu'à un effet du retournement de la conjoncture et à une transformation du cadre institutionnel, qui permet d'accompagner les mutations de la relation salariale, notamment dans les entreprises du marché primaire.

L'analyse des problèmes de santé en fin de carrière (voir tableau 1) montre en effet que, contre toute attente, le segment comportant le plus de risques est le segment primaire : toutes choses égales par ailleurs, un individu ayant travaillé sur ce segment présente quatorze fois plus de risque d'avoir un problème de santé en fin de carrière que s'il avait travaillé sur le segment secondaire. À partir des années 1970, les marchés internes connaissent en effet une transformation profonde de leur mode de fonctionnement. Sur ce segment, la crise gèle les embauches et fige les positions des travailleurs sur leur poste de travail. Puisqu'il n'est plus possible de se mettre à l'abri quand l'âge avance, les restrictions d'aptitude se multiplient dans les années 1970-1980: dans l'usine de carrosserie de Peugeot, $51 \%$ des ouvriers de l'atelier d'habillage-caisse ont au moins une restriction médicale ; 2 ou $3 \%$ du personnel se sont vu reconnaître une invalidité (Durand \& Hatzfeld, 2004). Les étrangers sont particulièrement concernés, car la plupart d'entre eux n'ont pas accès à la formation continue et aux voies de la mobilité ascendante.

Dans ce contexte, la mise en longue maladie ou en invalidité apparaît comme une forme concurrente des préretraites ou du chômage des travailleurs vieillissants; elle concerne essentiellement des personnes ayant une longue durée de séjour en France et des ouvriers restés longtemps sur le marché du travail. Les fins de carrière en longue maladie sont particulièrement nombreuses dans le secteur public, notamment dans les collectivités 
territoriales qui emploient un nombre non négligeable de salariés relevant du droit privé, employés par exemple à des emplois de nettoyage et de ramassage des déchets. Tel est le cas d'Azzedine Zghaier $^{9}$ : ce Tunisien arrivé en France en 1967, qui a travaillé comme ouvrier du bâtiment jusqu'en 1973 fait partie des rares ouvriers tunisiens du marché secondaire à avoir changé de segment. Il doit son maintien dans l'emploi à son embauche comme agent de voirie dans une mairie de banlieue parisienne, qui l'embauche jusqu'à sa retraite. Azzedine passe toutefois les trois dernières années de sa carrière en maladie : effet de l'usure lié à son emploi d'ouvrier nettoyeur, à son passé de manœuvre du bâtiment ? Peutêtre, mais en tout cas, s'il n'était pas parvenu à se reclasser chez un employeur du segment primaire, ses difficultés de santé n'auraient sans doute fait l'objet d'aucune prise en charge par le système de protection sociale.

Tableau 1 : probabilité de finir sa carrière dans une situation de non-emploi du fait d'un problème de santé (invalidité ou longue maladie) - régression logistique

\begin{tabular}{|c|c|c|c|c|}
\hline variables & coefficient & $\begin{array}{c}\text { écart- } \\
\text { type }\end{array}$ & significativité & $\begin{array}{l}\text { rapport } \\
\text { de } \\
\text { risques }\end{array}$ \\
\hline constante & $-0,567763$ & 1,24 & n.s. & \\
\hline $\begin{array}{l}\text { femme } \\
\text { homme de nationalité étrangère } \\
\text { homme de nationalité française }\end{array}$ & $\begin{array}{c}\text { ref. } \\
-0,042552 \\
-0,35059 \\
\end{array}$ & $\begin{array}{l}0,66 \\
0,59\end{array}$ & $\begin{array}{l}\text { n.s. } \\
\text { n.s. }\end{array}$ & \\
\hline $\begin{array}{l}\text { marié } \\
\text { autre situation matrimoniale }\end{array}$ & $\begin{array}{l}-0,092273 \\
\text { ref. }\end{array}$ & 0,48 & n.s. & \\
\hline $\begin{array}{l}\text { carrière erratique } \\
\text { carrière stagnante } \\
\text { carrière ascensionnelle } \\
\end{array}$ & $\begin{array}{l}1,592811 \\
1,715485 \\
\text { ref. } \\
\end{array}$ & $\begin{array}{l}0,75 \\
0,73\end{array}$ & $\begin{array}{l}* \\
*\end{array}$ & $\begin{array}{c}4,917551 \\
5,55937 \\
1 \\
\end{array}$ \\
\hline $\begin{array}{l}\text { moins de } 10 \text { ans sur le marché du } \\
\text { travail } \\
\text { de } 10 \text { à } 19 \text { ans sur le marché du travail } \\
\text { de } 20 \text { à } 29 \text { ans sur le marché du travail } \\
\text { plus de } 30 \text { ans sur le marché du travail } \\
\text { plus de } 30 \text { ans sur le marché du travail } \\
\text { moins de } 10 \text { ans sur le marché du } \\
\text { travail }\end{array}$ & $\begin{array}{c}-0,316328 \\
\text { ref. } \\
1,602274 \\
1,77619 \\
2,09 \\
\text { ref. }\end{array}$ & $\begin{array}{c}0,63 \\
0,7 \\
0,9\end{array}$ & n.s. & $\begin{array}{c}1 \\
4,964308 \\
5,907305 \\
8,084915 \\
1\end{array}$ \\
\hline $\begin{array}{l}\text { occupation d'un emploi ouvrier } \\
\text { jamais d'emploi ouvrier }\end{array}$ & $\begin{array}{c}1,406375 \\
\text { ref. }\end{array}$ & 0,56 & $*$ & $\begin{array}{c}4,081134 \\
1\end{array}$ \\
\hline $\begin{array}{l}\text { autre secteur } \\
\text { Bâtiment } \\
\text { bq assurces immob } \\
\text { commerce restauration } \\
\text { cstruction mecaq et electq } \\
\text { hygiène ménage } \\
\text { secteur inconnu } \\
\text { services publics }\end{array}$ & $\begin{array}{l}-3,629908 \\
-1,164243 \\
-0,52189 \\
-1,091281 \\
-2,938022 \\
-1,07594 \\
-0,196642 \\
-2,186898\end{array}$ & $\begin{array}{l}1,47 \\
1,09 \\
1,24 \\
0,79 \\
1,18 \\
1,05 \\
3,06 \\
1\end{array}$ & $\begin{array}{c}* \\
\text { n.s. } \\
\text { n.s. } \\
\text { n.s. } \\
* \\
\text { n.s. } \\
\text { n.s. } \\
*\end{array}$ & 0,112264 \\
\hline
\end{tabular}




\begin{tabular}{|c|c|c|c|c|}
\hline $\begin{array}{l}\text { textile cuir habillement } \\
\text { Transports }\end{array}$ & $\begin{array}{c}\text { ref. } \\
-1,362479\end{array}$ & 1,1 & n.s. & 1 \\
\hline cstruction mecaq et electq & ref. & & & \\
\hline bq assurces immob & 2,41 & 1,25 & $*$ & 11,13396 \\
\hline commerce restauration & 1,85 & 0,96 & $*$ & 6,35982 \\
\hline $\begin{array}{l}\text { commerce restauration } \\
\text { autre secteur }\end{array}$ & $\begin{array}{l}\text { ref. } \\
-2,54\end{array}$ & 1,35 & $*$ & $\begin{array}{c}1 \\
0,078866\end{array}$ \\
\hline $\begin{array}{l}\text { carrière sur le segment intermédiaire } \\
\text { carrière sur le segment intermédiaire } \\
\text { et primaire } \\
\text { carrière sur le segment intermédiaire } \\
\text { et secondaire } \\
\text { carrière sur le segment primaire } \\
\text { carrière sur le segment primaire et } \\
\text { secondaire }\end{array}$ & $\begin{array}{c}-1,492805 \\
-1,188986 \\
-2,798222 \\
\text { ref. } \\
0,269082\end{array}$ & $\begin{array}{l}0,78 \\
0,79 \\
1,39 \\
0,88\end{array}$ & $\begin{array}{c}* \\
\text { n.s. } \\
*\end{array}$ & $\begin{array}{c}0,060918 \\
1\end{array}$ \\
\hline $\begin{array}{l}\text { carrière sur le segment secondaire } \\
\text { carrière sur le segment primaire } \\
\text { carrière sur le segment primaire et } \\
\text { secondaire }\end{array}$ & $\begin{array}{l}\text { ref. } \\
2,68 \\
2,95\end{array}$ & $\begin{array}{l}1,02 \\
1,18\end{array}$ & $\begin{array}{l}* \\
*\end{array}$ & $\begin{array}{c}1 \\
14,58509 \\
19,10595\end{array}$ \\
\hline $\begin{array}{l}\text { cohorte } 1913-15 \\
\text { cohorte } 1923-25 \\
\text { cohorte } 1930-32 \\
\text { cohorte } 1936-38\end{array}$ & $\begin{array}{c}-1,1203 \\
-1,92899 \\
-1,467666 \\
\text { ref. }\end{array}$ & $\begin{array}{c}0,84 \\
0,7 \\
0,53\end{array}$ & $\begin{array}{c}\text { n.s. } \\
* \\
*\end{array}$ & $\begin{array}{l}0,145295 \\
0,230463\end{array}$ \\
\hline
\end{tabular}

Pour les travailleurs du segment secondaire, très majoritairement étrangers, la crise économique se traduit en effet par un autre mode de sortie du marché du travail, le retour au pays. Ces migrations de retour, qui empêchent de connaître l'état de santé de la population étudiée, concernent une proportion importante de la population étudiée : ce sont en effet un Tunisien sur trois de l'échantillon qui rentre de façon prématurée en Tunisie, mais un sur deux si l'on rapporte cette proportion à ceux qui ont travaillé sur le segment secondaire. Cet effet d'éviction rejoint les constatations faites par d'autres études portant sur la santé au travail des migrants, en particulier sur les mineurs marocains recrutés après une sélection drastique dans les années 1960, mais dont les contrats prenaient fin dès qu'ils présentaient un risque trop élevé de développer la silicose (Rosental \& Devinck, 2007).

Mal pris en charge par les dispositifs de protection sociale, mais soumis à des conditions de travail particulièrement dégradées et à la précarité de l'emploi, ces travailleurs du segment secondaire sont particulièrement nombreux à bénéficier d'une retraite pour inaptitude au travail. Pour les salariés de ces secteurs, usés par le travail, et à la carrière fragmentée, bénéficier d'une mise en inaptitude est souvent la seule façon d'obtenir une retraite à taux plein dès 60 ans. Conséquence de l'assouplissement des conditions d'accès à ce statut opéré par la réforme des retraites en 1971, la retraite au titre de l'inaptitude devient le refuge des populations interchangeables circulant sur le marché secondaire dont la carrière est extrêmement poreuse.

Ces deux échelles d'observation, par les fins de carrière et par le type de retraite, donnent ainsi deux images différentes de la santé des étrangers. Ce ne sont en effet pas les mêmes personnes qui finissent leur carrière en longue maladie et qui prennent leur retraite au titre de l'inaptitude médicale. II serait bien évidemment hasardeux d'en déduire que les 
problèmes de santé des premiers ont disparu au moment du passage à la retraite, tandis que les seconds auraient vu se développer des pathologies incapacitantes après leur fin d'activité. Ce phénomène traduit plutôt des modes différents de couverture sociale selon les segments du marché. Ainsi, les fins de carrière pour maladie ou invalidité apparaissent comme liées aux manifestations de la crise du marché primaire, dont les salariés sont relativement bien pris en charge par le système d'assurances sociales, qu'il s'agisse de l'assurance maladie, du chômage ou de la retraite; en revanche, dans les années 1980, les retraites pour inaptitude concernent avant tout d'anciens salariés du segment secondaire qui sont restés largement en marge du système de protection sociale.

La prise en compte du mode d'organisation du marché du travail dans l'analyse de la santé au travail permet ainsi de souligner l'importance des facteurs d'environnement de travail sur la santé et de mettre en regard les sorties d'emploi pour raisons de santé avec d'autres formes d'éviction du marché du travail. Avec la sur-sélection initiale, les sorties anticipées du marché du travail sont sans doute l'une des clés pour comprendre pourquoi les travailleurs étrangers, quoique soumis à des conditions de travail dégradées, ne connaissent pas des taux de mortalité plus élevés que la population française. Ces résultats soulignent aussi l'importance, pour analyser la santé au travail, de disposer d'éléments de description fine du marché du travail, trop souvent absents des enquêtes de santé.

Toutefois, les informations fournies par les dossiers de retraite décrivent tout autant un état de santé individuel que les inégalités d'accès à la couverture sociale et au marché du travail. Le poids du système de protection sociale représente un filtre difficilement dépassable dont il est indispensable de tenir compte pour qui souhaite analyser les liens entre état de santé des individus et conditions d'emploi par le biais des archives des caisses de retraite.

Ces limites invitent à développer les collaborations entre chercheurs en sciences sociales et médecins du travail pour coupler données médicales et données sociales, seules à même de permettre de mener à bien une véritable histoire des inégalités de santé et de faire sortir la santé des travailleurs étrangers de leur situation d'invisibilité relative.

\section{Références}

Al Zerdoumi M. (1952). Conditions de vie et d'hygiène des travailleurs nord-africains dans le département de la Seine, thèse de médecine, Paris, $(A M P, 1953)$ et " Le problème de la main-d'œuvre nord-africaine ", AMP, 1953, 14/6, 611-612.

Blanpain N. (2018), "L'espérance de vie par niveau de vie : chez les hommes treize ans d'écart entre les plus aisés et les plus modestes ", Insee Première, février, $n^{\circ} 1687$.

Bruno A.-S. (2004). "Inaptitude et immigration en France au XX $X^{\mathrm{e}}$ siècle ", in C. Omnes \& A.-S. Bruno (Eds.). Les mains inutiles. Inaptitude au travail et emploi en Europe. Paris: Belin, 125-145.

Bruno A.-S. (2010). Les chemins de la mobilité : Migrants de Tunisie et marché du travail parisien depuis 1956. Paris : Éditions de l'École des hautes études en sciences sociales. Colin M. (1953). La morbidité des Nord-Africains dans la métropole, AMP, 14/6, 594601.

Comité médical et médico-social d'aide aux migrants (1972). La santé des migrants. Paris : Société d'édition Droit \& Liberté. 
Cottereau A. (1983). Usure au travail. Destins masculins et destins féminins dans les cultures ouvrières en France, au XIX ${ }^{\mathrm{e}}$ siècle. Le Mouvement social, 124, 82-83.

Darmon N. \& Khlat M. (2001). An overview of the health status of migrants in France, in relation to their dietary practices. Public Health Nutr. 4/2, 163-72.

Durand J.-P. \& Hatzfeld N. (2004). Quand une question marginale devient centrale: les restrictions d'aptitude au quotidien dans l'usine Peugeot-Sochaux. In C. Omnès \& A.-S. Bruno (Eds.), Les mains inutiles. Inaptitude au travail et emploi en Europe. Paris : Belin, 391392.

Gentilini M., Brücker G. \& de Montvalon R. (1984). La santé des migrants, Paris, Rapport au ministère des Affaires Sociales et de la Solidarité nationale et au secrétaire d'État chargé de la Santé.

Goldberg M., Melchior M., Leclerc A. \& Lert F. (2003). Epidémiologie et déterminants sociaux des inégalités de santé. Revue d'Épidémiologie et de Santé Publique, 51, 381-401.

Guillemard, A.-M. (1993). Emploi, protection sociale et cycle de vie : résultats d'une comparaison internationale des dispositifs de sortie anticipée d'activité. Sociologie du travail, 3, 257-284.

Housset P., Jacquemet J.-P. \& Ronne G. (1965). Étude des problèmes posés par la main-d'œuvre étrangère dans une grande usine de la région parisienne. $A M P, 26 / 3,139-148$.

Jusot F., Silva J., Dourgnon P. et Sermet C. (2008). État de santé des populations immigrées en France. Document de travail de I'IRDES, juillet, $\mathrm{n}^{\circ} 14$.

Khlat M. \& Courbage Y. (1995). Mortalité des immigrés marocains en France, de 1979 à 1991. II-Les causes de décès. Population, 2, 447-472.

Khlat M., Sermet C. \& Laurier D. (1998). La morbidité dans les ménages originaires du Maghreb sur la base de l'enquête Santé de I'Insee, 1991-1992. Population, 6, 1155-1184.

Leclerc A., Fassin D., Grandjean H., Kaminski M., Lang T. (Eds.) (2000). Les inégalités sociales de santé. Paris : La Découverte-INSERM.

Minni C. \& Topiol A. (2004). Les entreprises face au vieillissement de leurs effectifs. Economie et Statistique, 368, 43-63.

Mizrahi A. \& Wait S. (1993). Accès aux soins et état de santé des populations immigrées. Rapport du CREDES, $\mathrm{n}^{\circ} 968$.

de Montvalon R. (1975). Profil d'une journée d'études. Migrations Santé. Bulletin du Comité médical et médico-social d'aide aux migrants, avril/3, 4.

de Montvalon R. (1976). La santé des migrants est-elle un domaine réservé ? Migrations Santé. Bulletin du Comité médical et médico-social d'aide aux migrants, 7, 8.

Omnès, C. (2006). Hommes et femmes face à la retraite pour inaptitude de 1945 à aujourd'hui. Retraite et société, 49/3, 77-97.

Perrin J. (1976). La prévention des accidents du travail et les travailleurs migrants. Revue de médecine du travail, 4/1, 41-43.

Pitti L. (2009). Du rôle des mouvements sociaux dans la prévention et la réparation des risques professionnels : le cas de Penarroya, 1971-1988. In C. Omnès \& L. Pitti (Eds.). Cultures du risque au travail et pratiques de prévention au XXe siècle. La France au regard des pays voisins. Rennes: Presses universitaires de Rennes, 217-232.

Pitti L. (2010). Experts "bruts" et médecins critiques. Ou comment la mise en débats des savoirs médicaux a modifié la définition du saturnisme en France durant les années 1970. Politix, 103-132. 
Rosental P.-A. \& Devinck J.-C. (2007). Statistique et mort industrielle. La fabrication du nombre de victimes de la silicose dans les houillères en France de 1946 à nos jours, Vingtième Siècle. Revue d'Histoire, 95, 75-91. 